\title{
APLICACIONES, FUNCIONAMIENTO Y REALIZACIONES DEL CAM PUS VIRTUEL FLE - UNIVERSITÉ DE LEÓN
}

\author{
Mario TOMÉ \\ Universidad de León
}

\section{Introducción}

El Campus Virtual FLE (Francés Lengua Extranjera) es un espacio pedagógico que forma parte del Proyecto FLEN ET (Français langue étrangère et Internet), alojado en el servidor web de la Universidad de Léon (dirección internet: http://www3.unileon.es/dp/dfm/flenet/), al tiempo que utiliza la plataforma BSCW (Basic Support for Cooperative Work) situada en la RedIRIS (Red académica y de investigación española), dentro del marco de Flenet RedIRIS (dirección internet: $h$ ttp://flenet.rediris.es). En la actualidad FLENET es un PROYECTO (I+D) DE INVESTIGACIÓN CIENTÍFICA Y DESARROLLO TECNOLÓGICO (2003-2006), adscrito al PROGRAMA NACIONAL DE PROMOCIÓN GENERAL DEL CONOCIMIENTO en el área de Filología y Filosofía (Referencia: BFF2003-06268).

Este dispositivo pedagógico de carácter híbrido (enseñanza presencial y a distancia) creado en el año 2000 va dirigido a los alumnos de la Universidad de León de los programas y asignaturas que se señalan más adelante, así como a docentes y alumnos de otros centros einstituciones (cursos de formación).

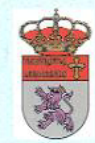

CAMPUS VIRTUEL - FLE Dpto. Filologia Moderna

Université de León

Inscription

Espaces de travail

La classe de

français

Espace public

Bibliothèque

Forum / Chat

Le site Flenet

La poste

Guide du BSCW

Outils

Univ. de León

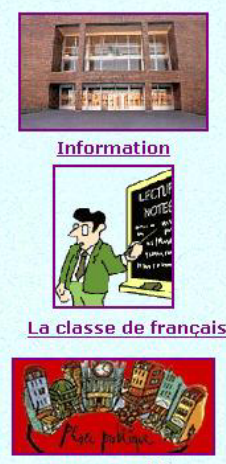

Espace public

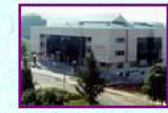

Bibliothèque

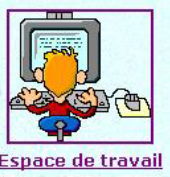

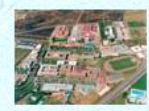

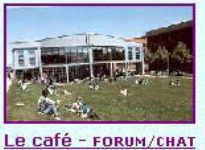

Le café - FORUM/CHAT
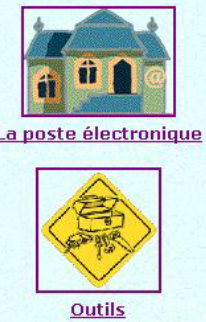
Podemos señalar los siguientes objetivos del Campus Virtuel FLE:

- Proporcionar recursos y actividades pedagógicas para alumnos y docentes dentro del campo del Francés Lengua Extranjera.

- Facilitar la ayuda, colaboración, tutorización y evaluación de los alumnos de las distintas asignaturas, programas y cursos de formación.

- Constituir un curso de enseñanza a distancia para los alumnos que no pueden asistir de manera presencial.

- Favorecer la formación de docentes y alumnos como laboratorio de experiencias pedagógicas en la aplicación de las Nuevas Tecnologías (TIC e Internet) a la enseñanza de una lengua extranjera.

Las asignaturas, programas y cursos de formación que se vienen desarroIlando hasta la actualidad con la ayuda del Campus Virtuel FLE son los siguientes, quedando definidos dentro de los parámetros:

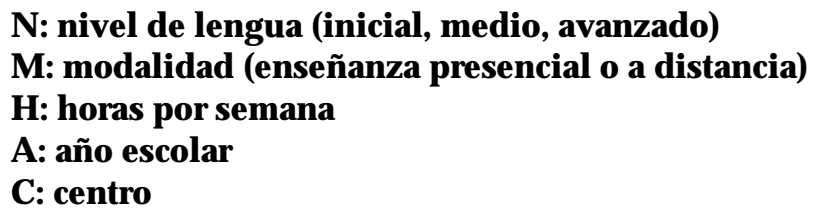

$2^{a}$ Lengua y su literatura Francés I (Filología Inglesa)

N : inicial; M : enseñanza presencial; H : 1; A: 2000-2001, 2001-2002, 20022003; 2003-2004 C : Universidad de León

\section{$2^{a}$ Lengua y su literatura Francés I (Filología Hispánica)}

N : medio; M : enseñanza presencial; H : 1; A: 2001-2002, 2002-2003 C: Universidad de León

\section{$2^{a}$ Lengua B: Francés (Lingüística)}

N : avanzado; $\mathbf{M}$ : enseñanza presencial; H : 1; A : 2001-2002, 2002-2003; 20032004 C : Universidad de León

Fonética francesa (Maestro en lengua extranjera)

N : avanzado; M : enseñanza presencial; H : 1; A : 2000-2001, 2001-2002, 20022003; 2003-2004 C : Universidad de León

Lengua extranjera y su didáctica II - Francés (Maestro en lengua extranjera) de León

N : avanzado; M : enseñanza presencial; H : 2; A : 2003-2004 C: Universidad

Las TICE aplicadas a la enseñanza de lenguas (Doctorado de Calidad)

$\mathbf{N}$ : en español; $\mathbf{M}$ : enseñanza presencial y a distancia; $\mathbf{H}$ : 1 crédito (10 horas); A : 2002-2003 C: Universidad de León 
Nuevas tecnologías: Metodologías y herramientas aplicadas a la enseñanza de lenguas (Doctorado "Interculturality and translation)

$\mathbf{N}$ : en español; $\mathbf{M}$ : enseñanza presencial y a distancia; $\mathbf{H}$ : 1,5 créditos (15 horas); A : 2003-2004 C : Universidad de León

\section{ciclo: \\ Cursos de formación o instrumentales para docentes o estudiantes de 3er}

Webpratique FLE (Curso a distancia RedIRIS)

N : avanzado M: a distancia H : 10 lecciones ; A : 2002-2003, 2003-2004; C: RedIRIS (plataforma BSCW)

Curso de formación para profesores de FLE: El ordenador y otros recursos en el aula de francés - Curso ACD-C. Centro de Profesores y de Recursos ZAMORA , 13-14 febrero, 2002

Curso de formación- Stage pédagogique: Phonétique française sur Internet pour le F.L.E. Centre Universitaire d' études françaises (C.U.E.F.) Université Stendhal-Grenoble 3. Stage pédagogique 29 juillet - 1er août 2002

Curso de formación para profesores de FLE: Didáctica de la lengua francesa en la ESO y Bachillerato. Consejería de Educación y Cultura / Junta de Castilla y León / Universidad de Salamanca. A vila, 25 febrero 2003.

Curso de formación para profesores de FLE: Novedades en literatura, fonética y gramática francesas. Estrategias y herramientas de aprendizaje. Consejería de Educación y Cultura / Junta de Castilla y León / Universidad de Salamanca. Zamora, 26 febrero 2003.

Curso de formación - Stage pédagogique: Ressources Internet pour la phonétique du F.L.E.Centre Universitaire d'études françaises (C.U.E.F.)Université Stendhal-Grenoble 3. Stage pédagogique. 26-30 juillet 2003.

Curso Instrumental: Nuevas tecnologías en la enseñanza de lenguas. Universidad de León. 29-30 septiembre 2003.

\section{Descripción del Campus Virtuel FLE}

El acceso al Campus Virtuel FLE se realiza a partir de la página web del mismo nombre en la Universidad de León (Véase: Anexo Imagen 1) (dirección internet: http://www3.unileon.es/dp/dfm/flenet/ BSCWprojet/index.html), la cual permite entrar en los distintos recursos (contenidos y actividades), espacios de trabajo y herramientas (correo, forum, chat, publicación web) que se detallan a continuación: 


\section{INFORMACIÓN}

El alumno se inicia en las posibilidades del Campus Virtuel FLE: inscripción, programa de las asignaturas, plan de trabajo, utilización del espacio de trabajo, guía de uso de la plataforma BSCW (Basic Support for Cooperative Work)

\section{BIBLIOTECA}

Facilita la consulta de numerosos documentos (páginas web, bases de datos, revistas, etc), así como también constituye una selección de recursos en relación con la lengua, la literatura y la cultura francesas.

\section{ESPACIOS DE COMUNICACIÓN}

Permiten desarrollar tareas docentes y de tutorización, así como el contacto entre alumnos.

Modalidades:

- Correo electrónico

- Forum de discusión y Chat en directo

- Plataforma de trabajo colaborativo BSCW

\section{LA CLASE DE FRANCÉS}

Es el centro de recursos para desarrollar las labores docentes, así como las actividades y tareas de los alumnos. Este espacio pedagógico se compone de los siguientes elementos:

1. Activités/Scénarios de PRÉSENTATION

2. Activités/Scénarios d' IN FORMATION / DEMANDE

3. Faire des exercices en ligne

PHONÉTIQUE

GRAM MAIRE

LEXIQUE

4. Tâches / Activités / Scénarios de recherche

$M$ anuel de grammaire

M anuel de prononciation

5. A nalyser des ressources FLE sur Internet

6. Scénarios pédagogiques

\section{EL ESPACIO DE TRABAJO}

Es el lugar donde los alumnos depositan sus trabajos y colaboraciones. Así se accede por la vía de la plataforma BSCW de RedIRIS al conjunto de carpetas y archivos que constituyen el historial y trabajo desarrollado por los alumnos. Observamos las siguientes secciones: 
Webpratique FLE - Cours à distance

A pprendre le français et découvrir Internet

http://cvu.rediris.es/pub/bscw.cgi/0/440552

Phonétique Française 2004

http://cou.rediris.es/pub/bscw.cgi/0/294294

Lengua extranjera y su didáctica II:

http://cou.rediris.es/pub/bscw.cgi/0/445781

$N$ uevas tecnologías en la enseñanza de lenguas:

Curso Instrumental 2003

http://cou.rediris.es/pub/bscw.cgi/0/429388

Páginas Web alumnos $h t t p: / / m e m b r e s . l y c o s . f r / u n i l e 2 /$

Las NTIC aplicadas a la enseñanza de lenguas:

D octorado de Calidad 2003: $h t t p: / /$ cou.rediris.es/pub/bscw. cgi/0/336832

Web al umnos de D octorado: $h t t p: / / m e m b r e s . l y c o s$. fr/doctorado/

\section{EL ESPACIO PÚBLICO}

Permite el acceso a las realizaciones y publicaciones Internet de los alumnos, entre las que destacamos:

\section{Docencia UNILEON - FLENET RedIRIS}

http://cou.rediris.es/pub/bscw.coi/0/253283

Journal 2004. Universidad de Leon:

http://www3.unileon.es/dp/dfm/flenet/courstourdumonde/carnet.htm

\section{Espacio público FLEN ET. RedIRIS:}

http:/ / cvu.rediris.es/ pub/ bscw.cgi/ 0/ 246617

Proyecto EURO PA UL 2004 (Intercambio y correspondecia entre clases): http://www.ac-versailles.fr/etabliss/plapie/Euro2004Accueil.htm

Realizaciones y resultados (2002-2004):

http://www3.unileon.es/dp/dfm/flenet/BSCWprojet/resultats.html

3. Funcionamiento y desarrollo de la docencia / tutorización en el Campus Virtuel FLE.

Las distintas unidades didácticas y tareas (tâches pédagogiques) que deben realizar los alumnos se publican en los diferentes espacios de trabajo asignados para cada materia o curso (Webpratique FLE, Phonétique Française 2004, etc.) o 
también pueden enviarse por correo electrónico a los alumnos a partir de una lista de difusión.

Cada unidad didáctica se organiza en torna a:

- Contenidos (consulta de documentos y recursos didácticos, elaborados por el docente o disponibles en Internet).

- Tareas, ejercicios y trabajos (realizar un itinerario programado por el docente, elaborar un proyecto, analizar recursos, crear un sitio internet, etc.) que implican actualizar y desarrollar competencias técnicas, lingüísticas y culturales.

Los alumnos depositan sus trabajos y colaboraciones en las carpetas personales asignadas dentro de la plataforma BSCW de Flenet RelRIS.

El profesor revisa el trabajo, otorgándole una calificación y añadiendo los comentarios y correcciones pertinentes, y decide su publicación en el ESPACIO PÚBLICO del Campus Virtuel FLE.

Los alumnos consultan los comentarios y evaluación del profesor, pudiendo añadir nuevos comentarios o preguntas. Para los trabajos en equipo, varios alumnos pueden enviar sus mensajes actualizando un debate / diálogo moderado por el profesor.

Las distintas unidades didácticas han sido elaboradas con la intención de conjugar los distintos componentes del dispositivo pedagógico ( recursos, tareas, espacios de comunicación, tutorización, etc.). Estas vienen definidas en base a criterios metodológicos como los que siguen: mixto.

LUG AR / CANAL: sala de ordenadores - curso a distancia por Internet -

NIVEL DE LEN GUA : inicial, medio, avanzado.

RELACIÓN PEDAG ÓGICA: presencial o a distancia; en autonomía, itinerario dirigido.

TIPO DE ACTIVIDAD: tareas dirigidas; búsqueda documental; realización de ejercicios orales o escritos; elaboración de trabajos individuales o en común; análisis de documentos y recursos en internet.

OBJETIV OS PED A G Ó GICOS: adquisición y práctica de la lengua francesa (oral y escrita); descubrir las posibilidades de comunicación y de información delnternet. 
CORRECCION / EVALUACIÓN : trabajos supervisados por el profesor en la plataforma BSCW; orientación, ayuda y corrección de problemas técnicos y lingüísticos

TUTORIZACIÓN : Alumnos y profesor se encuentran en horas presenciales fijadas, al tiempo que dial ogan a distancia por la vía de la plataforma BSCW o del correo electrónico.

\section{Realizaciones y resultados del Campus Virtuel FLE}

Teniendo en cuenta las tipologías de las tareas pedagógicas propuestas por F. Henri et K. Lundgren-Cayrol (1997) y F. Mangenot (2003) para los contextos de la Formación abierta y a distancia (FOAD) hemos agrupado las realizaciones de los alumnos en los siguientes apartados:

\subsection{La búsqueda colectiva}

La búsqueda guiada o dirigida por el docente puede tomar la forma de una investigación, un rallye o búsqueda de un tesoro. Generalmente se propone a los alumnos un cuestionario o ejercicios (comprensión, pronunciación, léxico, redaccción, etc.) dentro de un itinerario con direcciones internet para consultar o visitar

\section{Voyage virtuel León-Toulouse:}

http://www3.unileon.es/dp/dfm/flenet/courstourdumonde/culture.htm\#activités

Faire des exercices en ligne: Phonétique, G rammaire, Lexique: http://www3.unileon.es/dp/dfm/flenet/BSCWprojet/classe.html\#phonetique der:

WEBPRATIQUE - FLE Leçon no 1: Naviguer. Créer des favoris. Sauvegarhttp://www3.unileon.es/dp/dfm/flenet/courstourdumonde/webpratique.htm\#navig uer

WEBPRATIQUE - FLE Leçon no 4: Courrier. Inscription. Boîte aux lettres: http://www3.unileon.es/dp/dfm/flenet/courstourdumonde/webpratique htm\#courrier

Scénarios pédagogiques (LA CLASSE DE FRANÇAIS):

http://www3.unileon.es/dp/dfm/flenet/BSCWprojet/classe.html\#scenariospedagogi ques

\subsection{El análisis crítico}


El docente propone distintos recursos Internet (sitios, espacios pedagógicos, etc) que los alumnos deben describir y evaluar aplicando determinados criterios de análisis (grilles d'analyse).

A nalyser des ressources FLE sur Internet (LA CLASSE DE FRANÇAIS): http://www3.unileon.es/dp/dfm/flenet/BSCWprojet/classe.html\#analyser\%20resso $\underline{\text { urces }}$

WEBPRATIQUE - FLE Leçon no 2: Trier et évaluer les ressources: les grilles d'analyse:

http://cou.rediris.es/pub/bscw.cgi/0/336811

NA VIG UER (Curso de D octorado, Curso Instrumental :

http://www3.unileon.es/dp/dfm/flenet/docto/navegar.html

Véase asi mismo el capítulo: M éthodologies / G rilles d'analyse

http://www3.unileon.es/dp/dfm/flenet/grilles.html

\subsection{El debate}

En los espacios de tipo forum o chat los alumnos pueden entrar en discusión entre sí o bien con el profesor. El debate presenta también la variante de un trabajo en equipo y una puesta en comun (presencial o a distancia), así como desarrollarse en el marco de la tutorización dentro de la plataforma BSCW (diálogo entre docente y alumno: consulta, solución de dificultades, evaluación de tareas).

\section{ÇAIS):}

Activités/Scénarios d'information/demande (LA CLASSE DE FRANhttp://www3.unileon.es/dp/dfm/flenet/BSCWprojet/classe.html\#actinformation

WEBPRATIQUE - FLE Leçon no 5: Participer dans un forum / liste de discussion et dans un chat:

http://www3.unileon.es/dp/dfm/flenet/courstourdumonde/webpratique.htm\#l4

Possibilités pédagogiques du chat. Jeu: "Le sphynx et l'orthographe" : http://www3.unileon.es/dp/dfm/flenet/courstourdumonde/chatflenet.htm

\subsection{La toma de decisiones y la solución de problemas.}

Hemos agrupado aquí estos dos tipos de tareas ya que son complementarias en el marco de proyectos pedagógicos como la correspondencia escolar o la creación de una página web para la clase. Los alumnos tienen que resolver distintos problemas propuestos por el docente (navegar y encontrar recursos, bajar y utilizar programas, seleccionar y organizar la información, etc.) ya sea de mo- 
do individual o bien en equipo, lo cual implica una negociación y puesta en común.

\section{Creación de un sitio Internet (C urso de D octorado):}

http://membres.lycos.fr/doctorado/

\section{Creación de un sitio Internet (C urso Instrumental):}

http://membres.lycos.fr/unile2/

WEBPRATIQUE - FLE Leçon no 7: Publier des pages web:

http://www3.unileon.es/dp/dfm/flenet/courstourdumonde/Webpratique/pub1.html

\section{Projet Europaul 2001 (échange entre classes):}

http://www3.unileon.es/dp/dfm/flenet/Europaul/europaul2001.htm

\section{Projet Europaul 2002 (échange entre classes) :}

http://www3.unileon.es/dp/dfm/flenet/Europaul/europaul2002.htm

\section{Projet Europaul 2003 (échange entre classes):}

http://www.ac-versailles.fr/etabliss/plapie/Euro2003Accueil.htm

\section{Projet Europaul 2004 (échange entre classes):}

http://www.ac-versailles.fr/etabliss/plapie/Euro2004Accueil.htm

\subsection{La elaboración de recursos pedagógicos.}

En los cursos de formación para profesores o estudiantes de tercer ciclo se propone a menudo este tipo de tarea que exige un mínimo de competencias técnicas y metodológicas previas (grilles d'analyse, manejo de programas de creación de ejercicios autocorrectivos, edición de páginas web, etc.).

\section{Creación de un sitio Internet (C urso de D octorado):}

http://membres.lycos.fr/doctorado/

\section{Creación de un sitio Internet (Curso Instrumental):}

http:/ / membres.lycos.fr/ unile2/

Asimismo se pueden consultar otras realizaciones de los alumnos en los apartados siguientes del Campus V irtuel FLE:

Réalisations des étudiants. Curso 2001-2002 : 2a-Lengua y su Literatura. FRANCESI (F.Hispánica):

http://www3.unileon.es/dp/dfm/flenet/courstourdumonde/webprealisations.htm 
Projets Europaul 2001, 2002, 2003, 2004. 2a Lengua y su Literatura. FRANCESI (F.Inglesa):

http://www.ac-versailles.fr/etabliss/plapie/Euro2004Accueil.htm

Tâches Webpratique FLE Curso 2002-2003: 2a Lengua y su Literatura. FRANCESI (F.Hispánica) y 2ạ Lengua B FRANCES (L.Lingüística) :

http://cvu.rediris.es/pub/bscw.cgi/0/301220

Doctorado de Calidad (Universidad de León 2002-2003): Las NTIC aplicadas a la enseñanza de lenguas.

http://cou.rediris.es/pub/bscw.coi/0/336832

Curso Instrumental (Universidad de León 2003): Nuevas tecnologías en la enseñanza de lenguas:

http://cou.rediris.es/pub/bscw.cgi/0/429388

\section{Referencias y Bibliografía}

De Lièvre, B., Q uintin, J.-J. \& D epover, C. (2002). "Une expérience d'implantation d'activités organisées à distance au niveau universitaire". In Actes du 19ème colloque del'AIPU (Louvain-la-N euve). Consulté en décembre 2003:

http://ute.umh.ac.be/site_ute2/download.php?fd=ressources/publications/C050.pdf

De Lièvre, B., D epover, C., Q uintin, J., Decamps, S. (2002). Les technologies peuvent-elles être la source de pédagogies plus actives?, In Actes du colloque del'AIPU (Louvain-La-N euve). Consulté en novembre 2003: http://ute.umh.ac.be/site_ute2/download.php?fd=ressources/publications/C009.pdf

D emaizière, F. (2002) Dispositifs de l'apprentissage individualisé, Notes de l'intervention à l'université Paul Valéry, Montpellier 3, le 18 janvier 2002. Consulté en avril 2004: http://www.sigu7.jussieu.fr/AEM/montpellier.htm

Dillenbourg, P., Baker, M., Blaye, A \& \& O 'M alley, C. (1996). "The evolution of research on collaborative learning". In Spada, E. \& Reiman, P. (eds.) Learning in Humans and Machines:

Towards an Interdisciplinary Learning Science. pp. 189-211. Oxford : Elsevier. Consulté en octobre 2003: http://tecfa.unige.ch/tecfa/publicat/dil-papers-2/Dil.7.1.10.pdf

Furstenberg, G. (1997) "Scénarios d'exploitation pédagogique", REVUE Le Français dans le Monde, Col. Recherches et A pplications, no12, Juillet 1997, pp.64-75.

G authier, P. (2001) La dimension cachée de la e-formation, Thot/ Cursus. Nouvelles de la formation à distance. Consulté en octobre 2003:

http://thot.cursus.edu/rubrique.asp? no $=15961$ 
George, S. (2001). Apprentissage collectif à distance. SPLACH : un environnement informatique support d'une pédagogie de projet. Thèse de doctorat en informatique soutenue à l'université du Maine. Consulté en octobre 2003 (Résumé): http://groucho.univ-lemans.fr/lium/theses/resume.php? ref=George

G regoire, R ., R. Bracewell et T. Laferrière (1996) L'apport des nouvelles technologies de l'information et de la communication (NTIC) à l'apprentissage des élèves du primaire et du secondaire. Revue documentaire, Ottawa, Canada, Rescol. Consulté en octobre 2003:

http://www.fse.ulaval.ca/fac/tact/fr/html/apport/apport96.html

Henri, F. \& Lundgren-Cayrol, K. (1997). Apprentissage collaboratif à distance, téléconférence et télédiscussion. Montréal : LICEF. Consulté en octobre 2003:

http://www.licef.teluq.uquebec.ca/Bac/fiches/f48.htm

Henri, F. \& Lundgren-Cayrol, K. (2001). Apprentissage collaboratif à distance. Presses de l'université du Québec.

Lancien, T. (1997), Internet et l' enseignant: de l'information à la formation partagée, REVUE Le Français dans le Monde, Col. Recherches et Applications, nำ12, Juillet 1997 pp.116-122.

Lewis, R. (1998). "Apprendre conjointement : une analyse, quelques expériences et un cadre de travail". In Hypermédias et apprentissages 4. pp. 11-28. Paris : INRP et EPI.

M angenot, F.(1996) L'apprenant, l'enseignant et l'ordinateur: un nouveau triangle didactiqu ?, in actes du congrès "Linguaggi della formazione : l'informatica", organisé par I'IRRSAE (institut régional de recherche, d'expérimentation et de formation continue) du Val d'Aoste (Saint-Vincent, 5-6 sept. 1996). Consulté en novembre 2002: http://www.u-grenoble3.fr/espace_pedagogique/aoste.doc

Mangenot, F. (1999) UE/Module: TIC pour l'enseignement/apprentissage du FLE. Maîtrise de FLE. Cours Université Stendhal, Centre national d'enseignement à distance (Institut de Potiers-Futuroscope).

M angenot, F. \& M iguet, M. (2001). "Suivi par Internet d'un cours de maîtrise à distance: entre individualisation et mutualisation". In Hypermédias et apprentissages 5. pp. 259-266. Paris : INRP et EPI.

M angenot, F. (2003), Tâches et cooperation dans deux dispositifs universitaires de formation a distance. ALSIC, Université de Franche-Comté, Besançon, Vol. 6, Numéro 1, Juin 2003, pp. 109-125. Consulté en juin 2003:

http://alsic.u-strasbg.fr/Num10/mangenot/defaut.xml

Nunan, D. (1989). Designing Tasks for the Communicative Classroom. Cambridge University Press.

Oliver, D. \& Herrington, J. (2001). Teaching and Learning Online. Perth : Edith Cowan University.

O udart, P. (1997). Multimédia, réseaux et formation. Le français dans le monde, Recherches et applications, , numéro spécial, juillet 1997. Paris: HachetteEDICEF. 192pp. 
Tardif, J., Mukamurera, J. (1999). La pédagogie scolaire et les TIC: l'enseignement comme interactions, communication et pouvoirs, Revue Education et Francophonie: Les technologies de l'information et de la communication et leur avenir en éducation, Volume XXVII, No 2, automne-hiver. Consulté en juin 2003:

http://www.acelf.ca/reoue/XXVII-/articles/Tardif.html

Tomé, M . (2000). La communication et la recherche sur Internet dans l' enseignement du français langue étrangère, REVISTA "Estudios Humanísticos. Filología", no 22, Universidad de León, León, pp.175-194.

Tomé, M. (2001).Les ressources en FLE: La communication (II): Activités pédagogiques en communication. Thot / Cursus. Nouvelles de la formation à distance. Consulté en avril 2004: http://thot.cursus.edu/rubrique.asp?no=16394

Tomé, M . (2002). Analyse de la communication sur Internet en relation avec la didactique du Français langue étrangère, REVISTA "Estudios Humanísticos. Filología", no 24, Universidad de León, León, pp.341-360.

Tomé, M . (2003). WEBPRATIQUE - FLE. Apprendre le français, découvrir Internet, créer des pages web, Universidad de León. Secretariado de Publicaciones y Medios Audiovisuales, León, 110 pages. Consulté en mai 2004: http://www3.unileon.es/dp/dfm/flenet/livre2003.html

Warschauer, M. (2000). Online learning in second language classrooms: An ethnographic study. In M. Warschauer \& R. Kern (Eds.), Network-based language teaching: Concepts and practice (pp. 41-58). New York: Cambridge University Press.Consulté en octobre 2003: http://www.gse.uci.edu/markw/online-learning.html

Warschauer, M. (2003). Technology and equity: A comparative study. Paper presented at the Annual Meeting of the American Educational Research Association, Chicago. Consulté en janvier 2004:

http://www.gse.uci.edu/markw/tech-equity-aera.pdf. 